\title{
Fine Mapping of a New Race-Specific Blast Resistance Gene, Pi-hk2, in Japonica Heikezijing from Taihu Region of China
}

\author{
Wanwan He, Nengyan Fang, Ruisen Wang, Yunyu Wu, Guoying Zeng, Changhong Guan, Hao Chen, Ji Huang, \\ Jianfei Wang, Yongmei Bao, and Hongsheng Zhang
}

All authors: State Key Laboratory of Crop Genetics and Germplasm Enhancement, Jiangsu Collaborative Innovation Center for Modern Crop
Production, College of Agriculture, Nanjing Agricultural University, Nanjing 210095, China; fourth author: Lixiahe Agricultural Research
Institute of Jiangsu Province, Yangzhou 225007, China; and fifth author: Hunan Ava Seed Academy of Science, Changsha 410119, China. Accepted for publication 16 September 2016.

\begin{abstract}
Heikezijing, a japonica rice landrace from the Taihu region of China, exhibited broad-spectrum resistance to more than 300 isolates of the blast pathogen (Magnaporthe oryzae). In our previous research, we fine mapped a broad-spectrum resistance gene, Pi-hkl, in chromosome 11. In this research, 2010-9(G1), one of the predominant races of blast in the Taihu Lake region of China, was inoculated into 162 recombinant inbred lines (RIL) and two parents, Heikezijing and Suyunuo, for mapping the resistance-blast quantitative trait loci (QTL). Three QTL (Lsqtl4-1, Lsqtl9-1, and Lsqtl11-1) associated with lesion scores were detected on chromosomes 4, 9, and 11 and two QTL (Lnqtl1-1 and Lnqtl9-1) associated with average lesion numbers were detected on chromosomes 1 and 9 . The QTL Lsqtl9-1 conferring race-specific resistance to 2010-9(G1) at seedling

stages showed logarithm of the odds scores of 9.10 and phenotypic variance of $46.19 \%$ and might be a major QTL, named Pi-hk2. The line RIL84 with $P i-h k 2$ derived from a cross between Heikezijing and Suyunuo was selected as $P i-h k 2$ gene donor for developing fine mapping populations. According to the resistance evaluation of recombinants of three generations $\left(\mathrm{BC}_{1} \mathrm{~F}_{2}\right.$, $\mathrm{BC}_{1} \mathrm{~F}_{3}$, and $\left.\mathrm{BC}_{1} \mathrm{~F}_{4}\right), P i-h k 2$ was finally mapped to a $143-\mathrm{kb}$ region between ILP-19 and RM24048, and 18 candidate genes were predicted, including genes that encode pleiotropic drug resistance protein $4(n=2)$, WRKY74 $(n=1)$, cytochrome b5-like heme/steroid-binding domain containing protein $(n=1)$, protein kinase $(n=1)$, and ankyrin repeat family protein $(n=1)$. These results provide essential information for cloning of $P i-h k 2$ and its potential utility in breeding resistant rice cultivars by marker-assisted selection.
\end{abstract}

Rice blast, caused by the fungus Magnaporthe oryzae, is one of the most destructive diseases of rice. The annual loss of yield caused by rice blast was from 10 to $30 \%$, which was enough for feeding approximately 60 million people (Skamnioti and Gurr 2009). The application of resistance $(R)$ genes is considered as one of the most effective methods for minimizing crop losses caused by the disease (Fjellstrom et al. 2004). Developing rice cultivars with durable $R$ genes to blast is the most economically and environmentally friendly management approach to control rice blast disease (Wang et al. 1994). However, resistant cultivars often remain effective for only a few years because the pathogen $M$. oryzae has sufficient genetic flexibility to eventually overcome them (Dean et al. 2005; Skamnioti and Gurr 2009). Thus, one strategy of pyramiding multiple $R$ genes has been proposed as an alternative way for increasing the durability of resistance (Fjellstrom et al. 2004).

Over the past few years, approximately 100 rice $R$ genes have been mapped (Sharma et al. 2012), and at least 24 of $R$ genes have been cloned. Most of the $R$ genes are dominant and only pi21 and pi55 are recessive (Fukuoka et al. 2009; He et al. 2012). Some $R$ genes are clustered on chromosomes 6 and 11 (Chen et al. 2015), such as Pi9, Pi2, Piz-t, Pigm(t), and Pi50(t) on chromosome 6(Deng et al. 2006; Zhou et al. 2006; Zhu et al. 2012) and Piks, Pikh, Pikm, Pikp, Pik, Pil, Pi7(t), and Pi44(t), located near the telomeric end of the long arm of chromosome 11 (Ashikawa et al. 2008; Campbell et al. 2004; Chen et al. 1999; Hua et al. 2012; Yuan et al. 2011; Zhai

Corresponding authors: Y. Bao; E-mail address: yongmeibao@njau.edu.cn; and H. Zhang; E-mail address: hszhang@njau.edu.cn

*The $e$-Xtra logo stands for "electronic extra" and indicates that one supplementary table is published online.

(c) 2017 The American Phytopathological Society et al. 2011, 2014). Most $R$ genes encode nucleotide-binding site leucine-rich repeat (NBS-LRR) proteins, except for $P i$ - $d 2$, encoding a B-lectin receptor kinase (Chen et al. 2006) and resistance quantitative trait loci (QTL) gene pi21, encoding a proline-rich protein (Fukuoka et al. 2009). Among the 24 cloned genes, $\mathrm{Pi}$-ta (Lee et al. 2009a), Pi36 (Liu et al. 2007b) and Pit (Hayashi and Yoshida 2009) are reported as race-specific $R$ genes. The racespecific recognition of $R$ genes was usually determined by the conserved domains as the LRR region, the Toll/interleukin-1 receptor, and coiled-coil domains (Luck et al. 2000; Rairdan et al. 2008). In most cases, these conserved domains are involved in both pathogen detection and the activation of the downstream response (DeYoung and Innes 2006). Pi-ta, Pi36, and Pit trigger race-specific resistance through LRR domain recognition of the pathogen.

In our previous research, it was found that 'Heikezijing', a japonica rice landrace from the Taihu region of China, exhibited broad-spectrum resistance to at least 300 isolates. In total, 22 QTL conferring resistance to 17 isolates were identified in Heikezijing, and 5 QTL on chromosome 9 were effective against 13 isolates, with logarithm of the odds (LOD) scores of 2.65 to 14.57 and phenotypic variance of 7.43 to $68.61 \%$ (Shi et al. 2010; Wang et al. 2002). Main-effect locus qtl9-4-1 with the LOD score of 14.57 could explain $68.61 \%$ phenotypic variance against blast isolate JS02-95(ZE3). In this article, 2010-9(G1), one of the predominant isolates in the Taihu Lake region of China, was used to inoculate the mapping populations (recombinant inbred lines [RIL], $\mathrm{BC}_{1} \mathrm{~F}_{1}, \mathrm{BC}_{1} \mathrm{~F}_{2}$, $\mathrm{BC}_{1} \mathrm{~F}_{3}$, and $\mathrm{BC}_{1} \mathrm{~F}_{4}$ ) to fine map the race-specific $R$ gene $P i-h k 2$.

\section{MATERIALS AND METHODS}

Plant materials and growth. In all, $162 \mathrm{RIL}\left(\mathrm{F}_{2: 9}\right)$ derived from a cross between broad blast-resistant japonica landrace Heikezijing and blast-susceptible japonica landrace 'Suyunuo' were used to 
identify the resistant QTL to blast (M. oryzae). RIL84, selected from the RIL population and carrying few of the genomes from the resistance parent Heikezijing, was used as the target locus Pi-hk2 donor to develop the fine-mapping population. The $\mathrm{BC}_{1} \mathrm{~F}_{2}$ population with 1,560 plants, derived from a backcross of RIL84 and Suyunuo, was used for identifying recombinants in the target region with Pi-hk2.

Seed were sown in plastic trays ( 60 by 30 by $5 \mathrm{~cm}$ ) with sieved garden soil, as described by Wang et al. (2002). In total, 28 lines and two parents (Heikezijing and Suyunuo as the resistant and susceptible controls, respectively) were sown in each tray, and 10 seeds/ line were sown one den. Seedlings were grown in a greenhouse at 22 to $30^{\circ} \mathrm{C}$ with a light and dark cycle of 16 and $8 \mathrm{~h}$, respectively, until they were at the four-leaf stage for disease evaluation and DNA preparation. For expression analysis of candidate genes, the leaves of Heikezijing seedlings inoculated by blast isolate 2010-9(G1) were sampled at $0,4,12$, and $72 \mathrm{~h}$ after inoculation, frozen in liquid nitrogen immediately, and stored at $-80^{\circ} \mathrm{C}$.

Pathogen, inoculation, and disease evaluation. Isolate 2010-9 (G1), predominant in Jiangsu Province, China and provided by the Plant Protection Research Institute in Jiangsu Academy of Agricultural Sciences, was used for inoculating $162 \mathrm{~F}_{2: 9}$ RIL and two parents. Seven isolates belonging to different races, including 2009-70-1(A7), 2010-49-1(B1), 2010-28(C1), 2010-35-1(D13), 2011-40(E3), 2010-42-2(F1), and 2010-9(G1), were used to inoculate the $P i-h k 2$ donor RIL84 and the parents Heikezijing and Suyunuo.

Rice seedlings (four-leaf stage) were put into inoculation chambers and inoculated with a $40-\mathrm{ml}$ spore suspension $\left(5 \times 10^{4}\right.$ conidia $\left./ \mathrm{ml}\right)$, as in the method described by Wang et al. (2002). Each line was inoculated in two independent experiments, with three replications in each experiment. After inoculation, the seedlings were kept in the dark at $26^{\circ} \mathrm{C}$ with relative humidity of $95 \%$ for $24 \mathrm{~h}$, then transferred to a greenhouse with 25 to $28^{\circ} \mathrm{C}$ and $100 \%$ relative humidity, maintained by intermittently spraying water for 2 min every $3 \mathrm{~h}$.

Disease reactions were evaluated 1 week after inoculation with the pathogen. A disease rating system, as described by Mackill and
Bonman (1992) and Silué et al. (1992), was modified to determine the phenotypes of each progeny of the mapping population. Lesion scores from 0 to 5 were based on lesion type and lesion size, as described by Shi et al. (2010). For data analysis, the seedlings with rating 0,1 , and 2 were considered to be resistant and those rated as 3,4 , and 5 were considered to be susceptible.

Marker development and identification. Plant DNA was extracted from the 3-week-old seedlings using the sodium dodecyl sulfate method (Pich and Schubert 1993). For fine mapping the Pi$h k 2$ locus, three types of polymerase chain reaction (PCR)-based markers-simple-sequence repeat (SSR), intron length polymorphism (ILP), and insertion/deletion polymorphisms (InDel)-were developed in the target region with $P i$ - $h k 2$. SSR markers were directly adopted from the International Rice Microsatellite Initiative (http://www.gramene.org) (McCouch et al. 2002), and the SSR detection procedures followed the methods described by Zhu et al. (2004). ILP markers were designed around intronic regions which showed higher polymorphism than exonic regions and were developed according to predicted exons by software tools GENSCAN (http://genes.mit.edu/GENSCAN.html) and softberry (http://linux1.softberry.com/berry.phtml) (Wei et al. 2005). For InDel markers, sequence comparisons were developed between '93-11' and 'Nipponbare' in target regions by Pairwise BLAST (https://blast.ncbi.nlm.nih.gov/Blast.cgi) (Wu et al. 2013). All primers of these new markers were designed by Primer Premier 5.0.

QTL mapping. QTL analysis was performed using a previously constructed molecular linkage map (Shi et al. 2010). A genetic map of 162 RIL $_{2: 9}$ with a total distance of 2,457.16 centimorgans (cM) and an average distance of $17.68 \mathrm{cM}$ between two markers was constructed by MapMaker/EXP v. 3.0, as previously reported (Lander et al. 1987; Shi et al. 2010). QTL analyses were performed using the modified algorithm inclusive composite interval mapping ( $\mathrm{Li}$ and Ye 2007). The LOD threshold of 2.5 was used to detect QTL, as described by Lander and Kruglyak (1995). In this study, QTL detected by lesion number was labeled as LnqtlA-B and QTL detected by lesion score was labeled as LsqtlA-B,
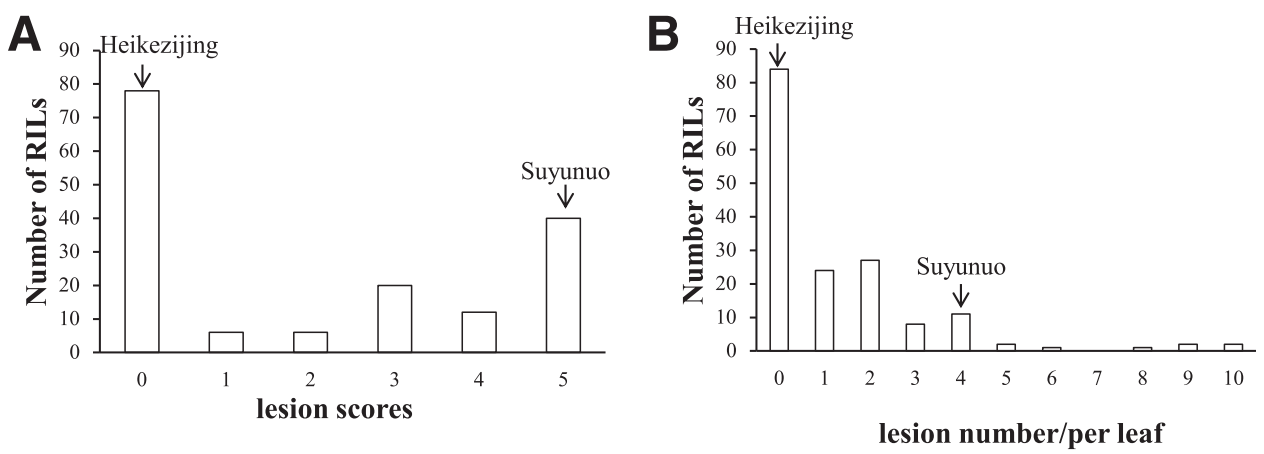

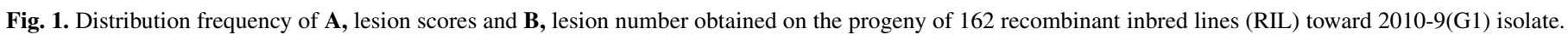
$\mathrm{X}$-axis is the trait of the lines and Y-axis is the number of lines with the same trait.

TABLE 1. Quantitative train loci (QTL) for resistance to 2010-9(G1) detected based on lesion score and lesion number

\begin{tabular}{|c|c|c|c|c|c|c|c|}
\hline Trait, QTL & Chromosome & Position $^{\mathrm{x}}$ & Left marker & Right marker & LOD $^{y}$ & $\operatorname{PVE}(\%)^{\mathrm{z}}$ & Add \\
\hline \multicolumn{8}{|l|}{ Lesion score } \\
\hline Lsqt14-1 & 4 & 63 & RM6089 & RM255 & 2.66 & 5.24 & -0.52 \\
\hline Lsqt19-1 & 9 & 287 & RM24097 & RM24019 & 9.10 & 46.19 & -1.62 \\
\hline Lsqt111-1 & 11 & 200 & RM27088 & RM27154 & 3.35 & 6.11 & -0.58 \\
\hline \multicolumn{8}{|c|}{ Lesion number } \\
\hline Lnqtl1-1 & 1 & 114 & RM5853 & RM1297 & 2.58 & 9.00 & -0.63 \\
\hline Lnqt19-1 & 9 & 26 & RM24097 & RM24019 & 5.94 & 14.10 & -0.79 \\
\hline
\end{tabular}

$x$ Position of peak logarithm of the odds (LOD) score.

y Peak LOD score in the QTL.

${ }^{\mathrm{z}}$ Explained the contribution to the phenotypic variance. 
as described by Shi et al. (2010), where $A$ indicates the chromosome number and $B$ represents the QTL.

Physical maps construction. In total, 1,560 $\mathrm{BC}_{1} \mathrm{~F}_{2}$ individuals were used for constructing a genetic linkage map and susceptible individuals were used for mapping $P i-h k 2$ by recessive class analysis methods. In all, $18 \mathrm{BC}_{1} \mathrm{~F}_{2}$ plants with a heterozygous region between RM24019 and RM24048 were used to generate $\mathrm{BC}_{1} \mathrm{~F}_{3}(3,360)$ and $21 \mathrm{BC}_{1} \mathrm{~F}_{3}$ plants with a heterozygous region between RM24019 and RM24048 were used to generate $\mathrm{BC}_{1} \mathrm{~F}_{4}$ $(4,160)$. In total, 3,360 $\mathrm{BC}_{1} \mathrm{~F}_{3}$ plants and $4,160 \mathrm{BC}_{1} \mathrm{~F}_{4}$ plants were used for genotyping and identifying recombinants between RM24019 and $\mathrm{RM} 24048$. The resistance phenotype of 13 recombinants obtained from $\mathrm{BC}_{1} \mathrm{~F}_{2}(n=4), \mathrm{BC}_{1} \mathrm{~F}_{3}(n=4)$, and $\mathrm{BC}_{1} \mathrm{~F}_{4}(n=5)$ were used to fine map the $P i-h k 2$. The physical map of the target region with $P i-h k 2$ can be subsequently constructed by BAC and PAC clones' sequences of Nipponbare in the International Rice Genome Sequencing Project (http://rgp.dna.affrc.go.jp/IRGSP/).

Candidate gene prediction and microarray data analysis. The sequences in the region of ILP-19 and RM24048 in BAC clone OJ1253E02 and PAC clone P0466E03 on chromosome 9 were downloaded from the RGP Web site. Open reading frames in the target region with $P i-h k 2$ were predicted by GENSCAN (http:// genes.mit.edu), FGENSH (http://linux1.softberry.com/), and Rice Genome Automated Annotation System (RiceGAAS) (http://rgp. dna.affrc.go.jp) software (Sakata et al. 2002).

The GENEVESTIGATOR (http://genevestigator.com/gv/) and Rice Oligonucleotide Array Database (http://www.ricearray.org/) were employed to analyze the expression of candidate genes based on 1,154 Affymetrix microarray datasets (http://www.ricearray.org/). The GENEVESTIGATOR was used to examine the expression patterns of candidate genes in all development stages and all organs.

The expression patterns of 18 candidate genes were detected by quantitative real-time PCR methods, as described by (Huang et al.
Chr. 9 LOD score

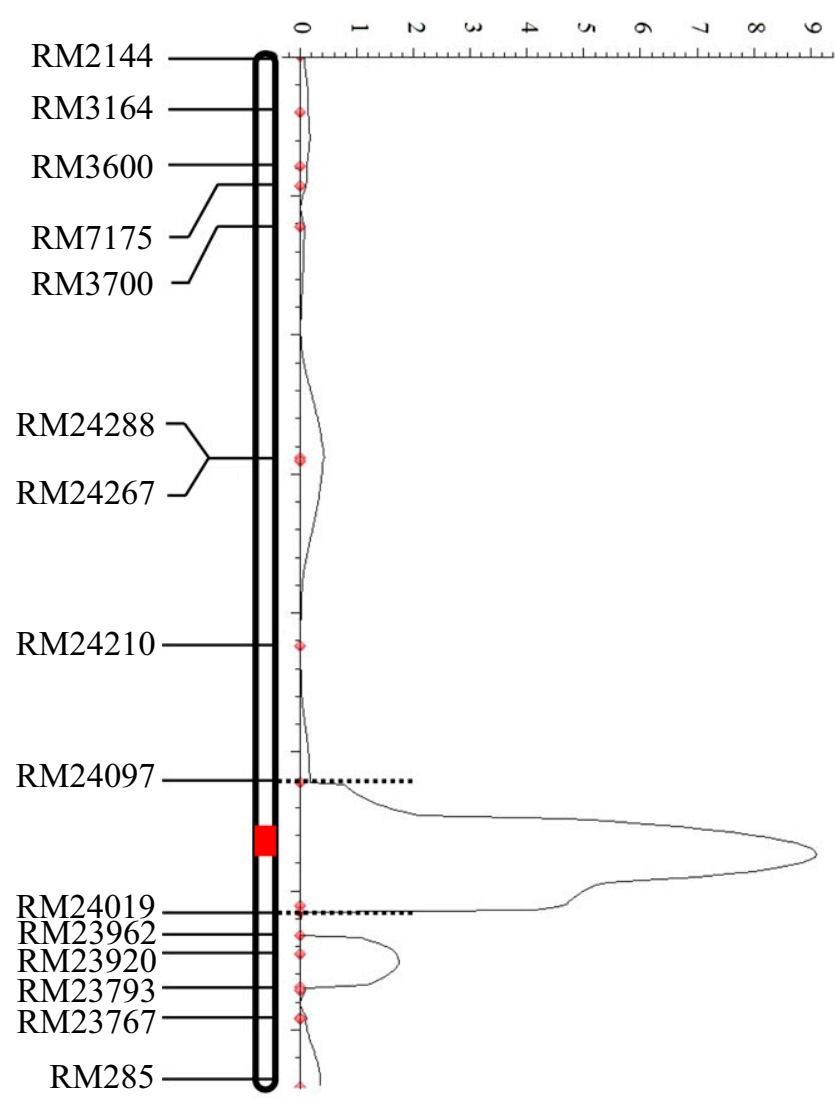

Fig. 3. Lsqtl9-1 for resistance to blast detected based on lesion score. LOD = logarithm of the odds and red location was Lsqtl9-1 position.
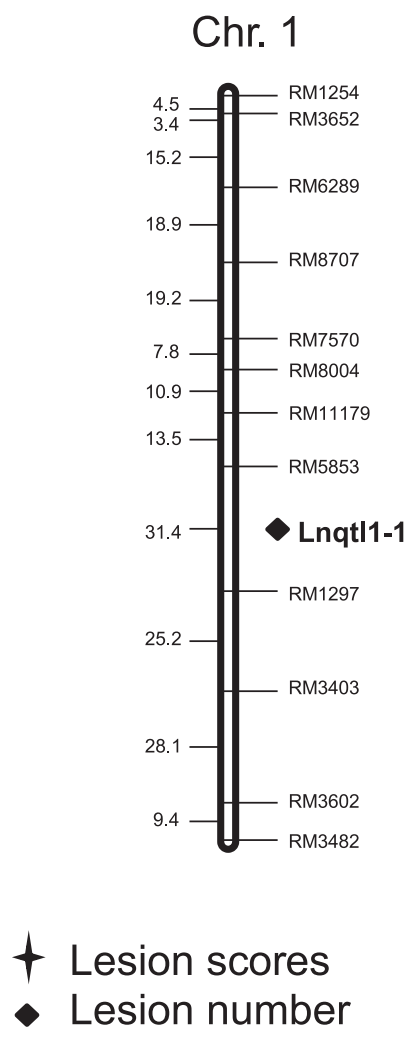

Chr. 4

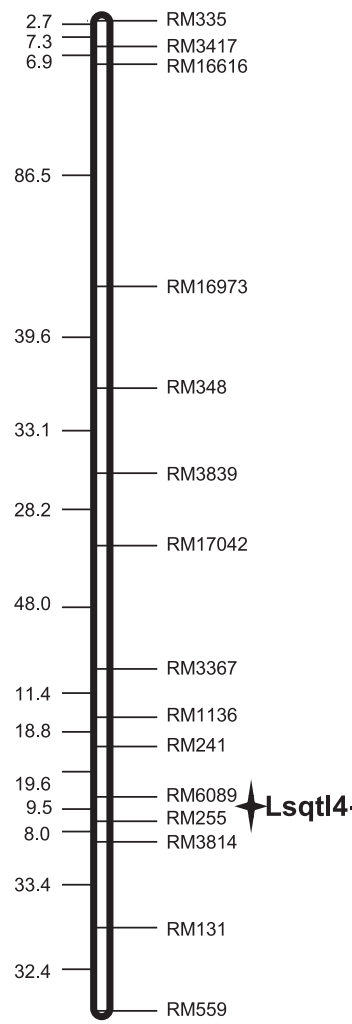

Chr. 9

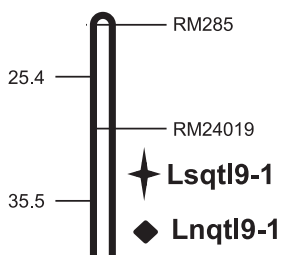

Chr. 11

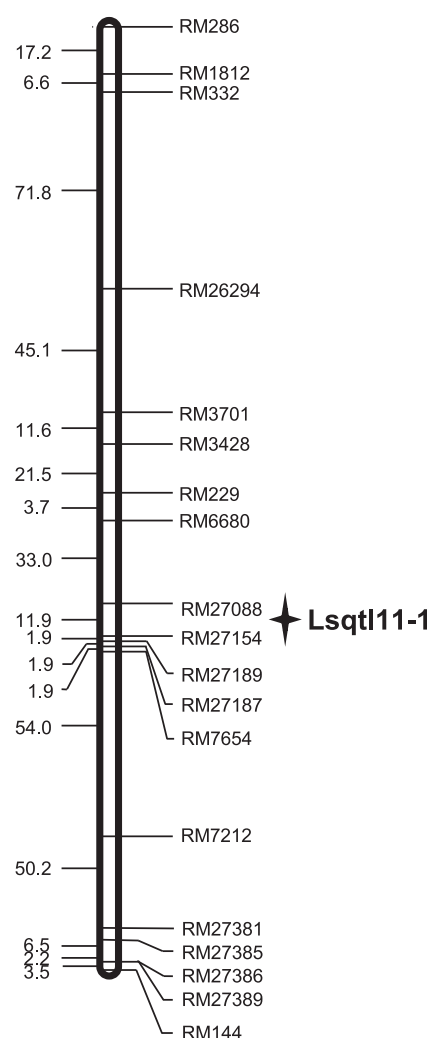

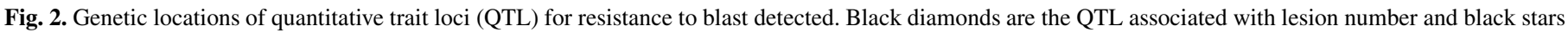
are the QTL associated with lesion scores. 
2008). The fold change in cDNA (target candidate gene) relative to the reference gene (18s-rRNA) was determined by the comparative cycle threshold method (Livak and Schmittgen 2001). Relative quantification relates the PCR signal of the target transcript in inoculated rice seedlings to that of uninoculated rice plants. Relative expression levels were calculated by the $2^{-\Delta \Delta C T}$ method (Livak and Schmittgen 2001). All reactions were performed in triplicate. The primers for 18 candidate genes and 18s-rRNA in quantitative realtime PCR assay are shown in Supplementary Table S1.

\section{RESULTS}

Race-specific QTL Lsqtl9-1 was identified. Lesion scores and lesion numbers of 162 RIL $\left(\mathrm{F}_{2: 9}\right)$ and two parents (Heikezijing and Suyunuo) inoculated with isolate 2010-9(G1) were evaluated. The two traits showed continuous frequency distributions among RIL populations (Fig. 1), indicating polygenic and quantitative resistance to the blast in resistant parent Heikezijing.

Five QTL were detected to be associated with lesion scores and lesion number, which were distributed on chromosomes 1, 4, 9, and

TABLE 2. Responses of Heikezijing, Suyunuo, and RIL84 to seven isolates ${ }^{z}$

\begin{tabular}{lcll}
\hline Isolate & Heikezijing & Suyunuo & \multicolumn{1}{c}{ RIL84 } \\
\hline $2009-70-1(\mathrm{~A} 7)$ & $0(\mathrm{R})$ & $2.5(\mathrm{MS})$ & $2.5(\mathrm{MS})$ \\
$2010-49-1(\mathrm{~B} 1)$ & $0(\mathrm{R})$ & $5(\mathrm{~S})$ & $5(\mathrm{~S})$ \\
$2010-28(\mathrm{C} 1)$ & $0(\mathrm{R})$ & $5(\mathrm{~S})$ & $5(\mathrm{~S})$ \\
$2010-35-1(\mathrm{D} 13)$ & $0(\mathrm{R})$ & $5(\mathrm{~S})$ & $3(\mathrm{~S})$ \\
$2011-40(\mathrm{E} 3)$ & $0(\mathrm{R})$ & $4(\mathrm{~S})$ & $5(\mathrm{~S})$ \\
$2010-42-2(\mathrm{~F} 1)$ & $0(\mathrm{R})$ & $5(\mathrm{~S})$ & $5(\mathrm{~S})$ \\
$2010-9(\mathrm{G} 1)$ & $0(\mathrm{R})$ & $5(\mathrm{~S})$ & $0(\mathrm{R})$
\end{tabular}

$\overline{\mathrm{z}} \mathrm{R}, \mathrm{MR}, \mathrm{S}$, and MS represent resistant, moderately resistant, susceptible, and moderately susceptible, respectively.
11 (Table 1; Fig. 2). Among them, Lsqtl4-1, Lsqtl9-1, and Lsqtl11-1, associated with lesion scores, were distributed on chromosomes 4 , 9, and 11, with LOD scores of 2.66, 9.10, and 3.35 and phenotypic variance of 5.24, 46.19, and 6.11\%, respectively. The two QTL Lnqtl1-1 and Lnqtl9-1 associated with average lesion number were distributed on chromosomes 1 and 9, with LOD scores of 2.58 and 5.94 and phenotypic variance of 9.00 and $14.10 \%$, respectively. The QTL Lsqtl9-1, with LOD score of 9.10 and phenotypic variance $46.19 \%$, was mapped between the SSR markers RM24019 and RM24097 on chromosome 9 (Fig. 3), which might be a major resistance QTL, and named as $P i-h k 2$.

The RIL populations were further inoculated by seven representative isolates: 2009-70-1(A7), 2010-49-1(B1), 2010-28(C1), 2010-35-1(D13), 2011-40(E3), 2010-42-2(F1), and 2010-9(G1). RIL84 conferring Pi$h k 2$ showed resistance to $2010-9(\mathrm{G} 1)$ but significant susceptibility to the other six isolates (Table 2). This result indicated that Pi-hk2 confers race-specific resistance to 2010-9(G1) at seedling stages.

Fine mapping of $\boldsymbol{P} \boldsymbol{i}$ - $h \boldsymbol{k} \boldsymbol{2}$. In total, 62 plants $\left(\mathrm{BC}_{1} \mathrm{~F}_{1}\right)$ derived from backcrossing RIL84 and Suyunuo were inoculated with isolate 2010-9(G1) in the growth chamber; of these, 33 individuals were resistant to 2010-9(G1) and 29 were susceptible. The numbers of resistant and susceptible plants fit a $1: 1$ ratio $\left(\chi^{2}=0.25<\chi^{2}{ }_{0.05}=\right.$ $3.84)$, suggesting that the resistance of RIL84 to isolate 2010-9(G1) was controlled by a single dominant gene.

To fine map gene $P i-h k 2$, four additional markers (RM24048, RM24065, RM7390, and RM3912) between RM24019 and RM24097 were selected that showed polymorphisms between parents Heikezijng and Suyunuo. In all, 1,560 $\mathrm{BC}_{1} \mathrm{~F}_{2}$ plants from $33 \mathrm{BC}_{1} \mathrm{~F}_{1^{-}}$ resistant plants were genotyped by those markers, and 12, 8, 5, 16, and 8 recombinants were identified by RM24019, RM24048, RM24065, RM7390, and RM3912, respectively. Through phenotype assays of the recombinants, $P i$-hk2 was mapped in the region of RM24019 and RM24048 with a physical distance of $520 \mathrm{~kb}$ (Fig. 4A).

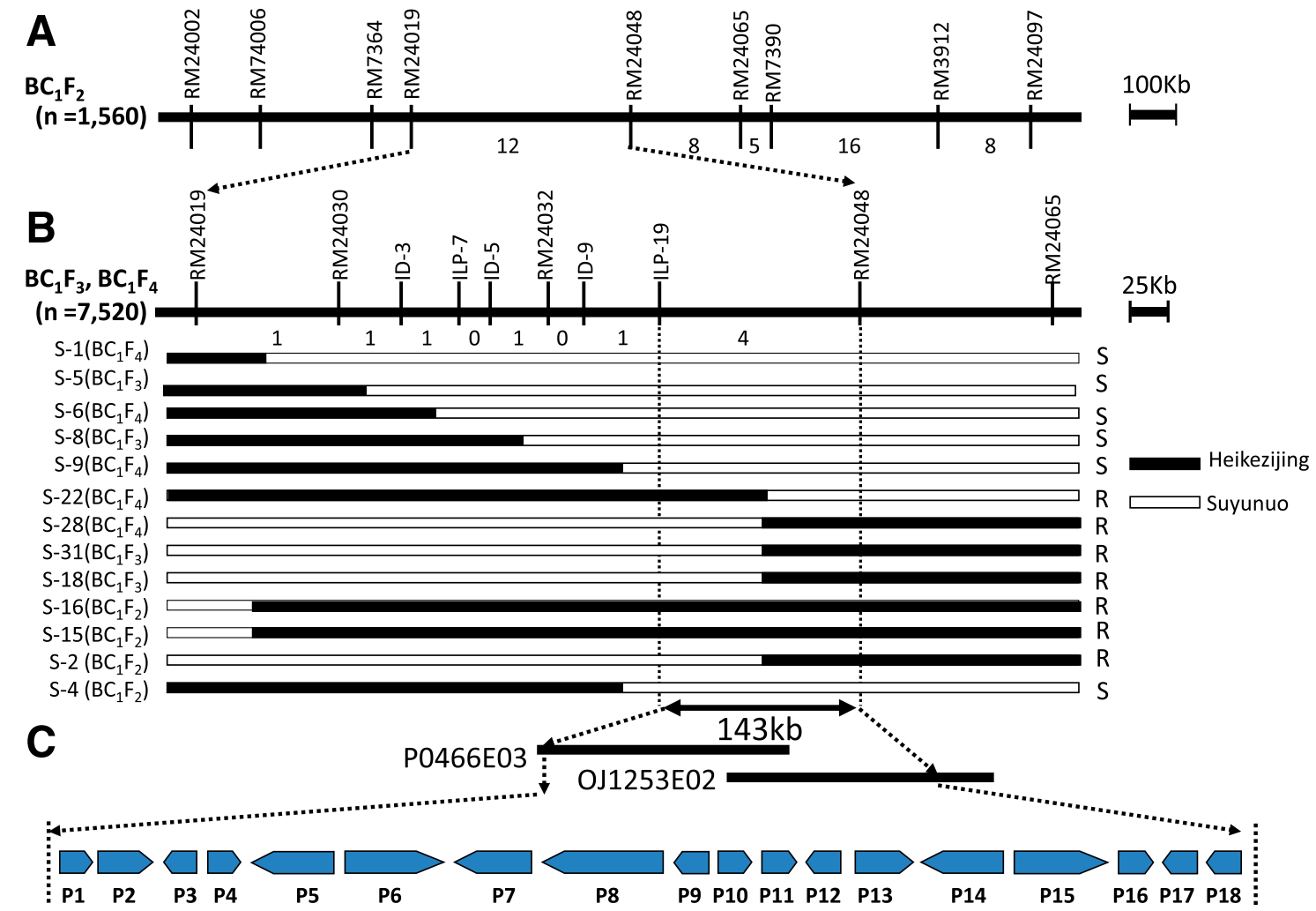

Fig. 4. Genetic and physical maps of the region of the $P i$ - $h k 2$ locus. A, Fine linkage map generated by analyzing $1,560 \mathrm{BC}_{1} \mathrm{~F}_{2} \mathrm{Segregating}$ plants. Recombinant numbers are given between markers. B, Physical map of $P i-h k 2$, constructed from the Nipponbare genomic sequence. Numbers below the map are recombinant numbers. C, Counting map and candidate genes of the Pi-hk2 locus. Short horizontal lines represent BAC/PAC clones of Nipponbare. These clones are anchored by the corresponding markers linked to the $P i$ - $h k 2$ locus. Arrows represent open reading frames predicted in the $P i$ - $h k 2$ region by RiceGAAS. 
To evaluate their resistance phenotype, 13 recombinant plants identified between RM24019 and RM24048 were inoculated with 2010-9(G1). For further delimitation of the $P i-h k 2$ locus, seven new markers were explored between RM24019 and RM24048 on the basis of sequences in a public database to determine the genotypes (Table 3). As a consequence of fine mapping, $P i$ - $h k 2$ was located in the region of ILP-19 and RM24048, which was $143 \mathrm{~kb}$ on physical map of the target region (Fig. 4B).

Candidate gene prediction and expression analysis. The target $143-\mathrm{kb}$ region $(10,021,585$ to $10,166,709)$ conferring Pi-hk2 in the Nipponbare sequence was covered by two BAC/PAC clones (OJ1253E02 and P0466E03), and 18 putative genes were predicted by GENSCAN, FGENSH, and RiceGAAS software (Fig. 4C). Among these 18 candidate genes $(P 1$ to $P 18), 6$ putative genes $(P 7, P 8, P 13$,
$P 14, P 16$, and $P 17$ ) might encode functional proteins, including $P 7$ and $P 8$ encoding pleiotropic drug resistance protein $4, P 13$ encoding WRKY74, P14 encoding cytochrome b5-like heme/steroid-binding domain containing protein, $P 16$ encoding protein kinase, and $P 17$ encoding ankyrin repeat family protein. Five genes $(P 1, P 3, P 12, P 9$, and $P 18)$ might encode hypothetical proteins and seven genes $(P 2, P 4$, $P 5, P 6, P 10, P 11$, and $P 15)$ encode transposon/retrotransposon proteins (Table 4).

The expression profiles of these candidate genes in various rice tissues were investigated based on microarray data deposited in the GENEVESTIGATOR. The expression of 12 candidate genes could be detected in rice culm (stem), sheath, root, panicle, leaf, pollen, inflorescence, node, embryo, and endosperm (Fig. 5). The expression of $P 1, P 3, P 5, P 11, P 16, P 17$, and $P 18$ showed higher expression

TABLE 3. Information on polymerase chain reaction-based markers used for mapping of $P i$ - $h k 2$

\begin{tabular}{|c|c|c|c|c|c|}
\hline Marker name & Marker type ${ }^{\mathrm{x}}$ & Primer sequence $\left(5^{\prime}-3^{\prime}\right)$ & Product size (bp) expected & Temp $\left({ }^{\circ} \mathrm{C}\right)^{\mathrm{y}}$ & $\mathrm{Gel}^{\mathrm{z}}$ \\
\hline RM24019 & SSR & $\begin{array}{l}\text { F:GGACAAGATGATGACACTCTGTTACC } \\
\text { R:TGCTTCTTAGTTCTCAGCCACACC }\end{array}$ & 84 & 55 & A \\
\hline RM24030 & SSR & $\begin{array}{l}\text { F:GTATGCCATATCCAACGACAATCC } \\
\text { R:GTGTGATTCTGATTGGCTGAAAGG }\end{array}$ & 82 & 55 & A \\
\hline ID-3 & InDel & $\begin{array}{l}\text { F:CGGTCAATACATCATCTCCTTCCTC } \\
\text { R:CTGCCATGACGTTCCAAGC }\end{array}$ & 714 & 54 & $\mathrm{C}$ \\
\hline ILP-7 & ILP & $\begin{array}{l}\text { F:CCCAAATTCCAGTCACCGCCGTCCG } \\
\text { R:CGCGCGATAAATGGTCGCCGGCAGC }\end{array}$ & 179 & 59 & A \\
\hline ID-5 & InDel & $\begin{array}{l}\text { F:TCTCAAGCATTACGATGACAGCCTT } \\
\text { R:AGGGCTGGGAACATTGGTATGGTTT }\end{array}$ & 1,245 & 60 & $\mathrm{C}$ \\
\hline RM24032 & SSR & $\begin{array}{l}\text { F:ATTCAACATGGGCTGCCTGTCC } \\
\text { R:GCTGCAGTCTGAATGGTGGTACG }\end{array}$ & 239 & 52 & B \\
\hline ID-9 & InDel & $\begin{array}{l}\text { F:AATCTACTTTCAGATGGTTTGGTCC } \\
\text { R:GCTAGTCTTAACGACATACTTGACG }\end{array}$ & 867 & 55 & $\mathrm{C}$ \\
\hline ILP-19 & ILP & $\begin{array}{l}\text { F:GACGACGGGGAGGCGGGATC } \\
\text { R:AGGAGGGCCGCCCAAGCAC }\end{array}$ & 151 & 52 & A \\
\hline RM24048 & SSR & $\begin{array}{l}\text { F:CTAGCTTCGTTCAAATCACGTAGG } \\
\text { R:GGATAGATGGATGGATGGATGG }\end{array}$ & 158 & 55 & A \\
\hline RM24065 & SSR & $\begin{array}{l}\text { F:TGCAGAGACAAGATAGGAATGC } \\
\text { R:CATGCATGTCAATGTGATCG }\end{array}$ & 145 & 52 & A \\
\hline RM7390 & SSR & $\begin{array}{l}\text { F:TGAGAGCTCGTAGGAAGTGTCC } \\
\text { R:CAGAGTCAGCAATCGCTAAGG }\end{array}$ & 244 & 52 & B \\
\hline RM3912 & SSR & $\begin{array}{l}\text { F:CACTCAGATTTGGCCGATCC } \\
\text { R:GCTGATCCAGATCTACCTGACACC }\end{array}$ & 263 & 58 & B \\
\hline RM24097 & SSR & $\begin{array}{l}\text { F:GTCCTTCGTCCACGGCAACTACG } \\
\text { R:TACGCCGAGGTACAGGTCGAAGC }\end{array}$ & 82 & 55 & A \\
\hline
\end{tabular}

x $\mathrm{SSR}=$ simple-sequence repeat $(\mathrm{SSR})$, InDel $=$ insertion/deletion polymorphisms, and ILP = intron length polymorphism.

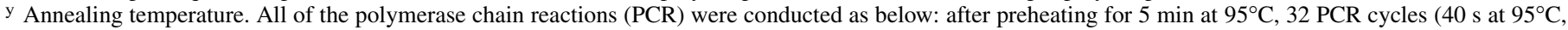
$40 \mathrm{~s}$ at the corresponding temperature described above, and $40 \mathrm{~s}$ to $2 \mathrm{~min}$ at $72^{\circ} \mathrm{C}$ ), followed by $10 \mathrm{~min}$ at $72^{\circ} \mathrm{C}$.

z $\mathrm{A}=8 \%$ acrylamide, $\mathrm{B}=6 \%$ acrylamide, and $\mathrm{C}=2 \%$ agarose.

TABLE 4. Candidate genes at the Pi- $h k 2$ region in Heikezijing

\begin{tabular}{|c|c|c|c|}
\hline Number & Annotated genes & Prediction of protein function ${ }^{y}$ & $N^{\mathrm{z}}$ \\
\hline 1 & LOC_Os09g16390 & Expressed protein & 171 \\
\hline 2 & LOC_Os09g16400 & TP, putative, CACTA, En/Spm subclass, expressed & 336 \\
\hline 3 & LOC_Os09g16410 & Expressed protein & 149 \\
\hline 4 & LOC_Os09g16420 & RP, putative, Ty3-gypsy subclass, expressed & 197 \\
\hline 5 & LOC_Os09g16430 & RP, putative, Ty3-gypsy subclass, expressed & 1,262 \\
\hline 6 & LOC_Os09g16440 & $\mathrm{TP}$, putative, unclassified, expressed & 722 \\
\hline 7 & LOC_Os09g16449 & Pleiotropic drug resistance protein 4 , putative, expressed & 479 \\
\hline 8 & LOC_Os09g16458 & Pleiotropic drug resistance protein 4 , putative, expressed & 423 \\
\hline 9 & LOC_Os09g16470 & Hypothetical protein & 68 \\
\hline 10 & LOC_Os09g16480 & $\mathrm{RP}$, putative, centromere-specific & 168 \\
\hline 11 & LOC_Os09g16490 & $\mathrm{RP}$, putative, unclassified & 209 \\
\hline 12 & LOC_Os09g16500 & Expressed protein & 107 \\
\hline 13 & LOC_Os09g16510 & WRKY74, expressed & 362 \\
\hline 14 & LOC_Os09g16520 & $\begin{array}{l}\text { Cytochrome b5-like heme/steroid-binding domain containing protein, } \\
\text { expressed protein }\end{array}$ & 119 \\
\hline 15 & LOC_Os09g16530 & TP, putative, unclassified, expressed & 1,586 \\
\hline 16 & LOC_Os09g16540 & Protein kinase, putative, expressed & 724 \\
\hline 17 & LOC_Os09g16550 & Ankyrin repeat family protein, putative, expressed & 688 \\
\hline 18 & LOC_Os09g16560 & hypothetical protein & 160 \\
\hline
\end{tabular}

y $\mathrm{TP}=$ transposon protein and $\mathrm{RP}=$ retrotransposon protein.

z Number of amino acids. 
levels in pollen. $P 7$ and $P 8$ were found to show a higher expression level in sheath; $P 12$ in endosperm and pollen; $P 13$ in inflorescence, leaf, and root; and P14 in panicle and culm (stem).

Real-time reverse-transcription PCR was performed to examine the expression pattern of candidate genes in Heikezijing seedlings induced by $2010-9$ (G1). The results showed that $P 11, P 13$, and $P 18$ were dramatically more highly expressed in Heikezijing, and were induced by inoculation. Among those candidate genes, the expression of $P 13$ was the highest; the other candidate genes were expressed relatively less either with or without inoculation (Fig. 6). Genes P1, P3, P4, P5, $P 6, P 9$, and $P 15$ were not expressed before or after inoculation. According the structure and expression of candidate genes, two genes (P13 and P18) might be the candidates of gene Pi-hk2.

\section{DISCUSSION}

It was reported that there were some broad-spectrum or durably resistant cultivars such as 'Moroberekan', 'Suweon', 'Teqing', 'Sanhuangzhan 2', 'GuMei 2', and 'XZ3150' (Ma et al. 2015). Several $R$ genes have been identified from Moroberekan (Jeon et al. 2003), 'Suweon 365' (Ahn et al. 2000), Teqing (Tabien et al. 2000), and Sanhuangzhan 2 (Liu et al. 2004). Heikezijing, one japonica rice landrace from the Taihu region of China, exhibited broadspectrum resistance to at least 300 isolates belonging to seven Chinese blast races (Shi et al. 2010; Wang et al. 2002). In our previous research, a broad-spectrum $R$ gene, $P i-h k l$, has been identified on chromosome 11 of Heikezijing. In this article, we fine mapped race-specific $R$ gene $P i$ - $h k 2$ on chromosome 9 .

Race-specific resistance closely follows the classical gene-forgene relationship. The isolation and subsequent characterization of $R$ genes will help to unravel the molecular mechanisms underlying the interaction between host and pathogen. To date, five pairs of avirulence $(A v r)$ and $R$ genes (AvrPita versus $P i$-ta, Avr-Pik versus Pik, AvrPiz-t versus Piz-t, Avr-Pia versus Pia, and Avrl-CO39 versus $P i$-CO39) have been intensively studied in rice blast

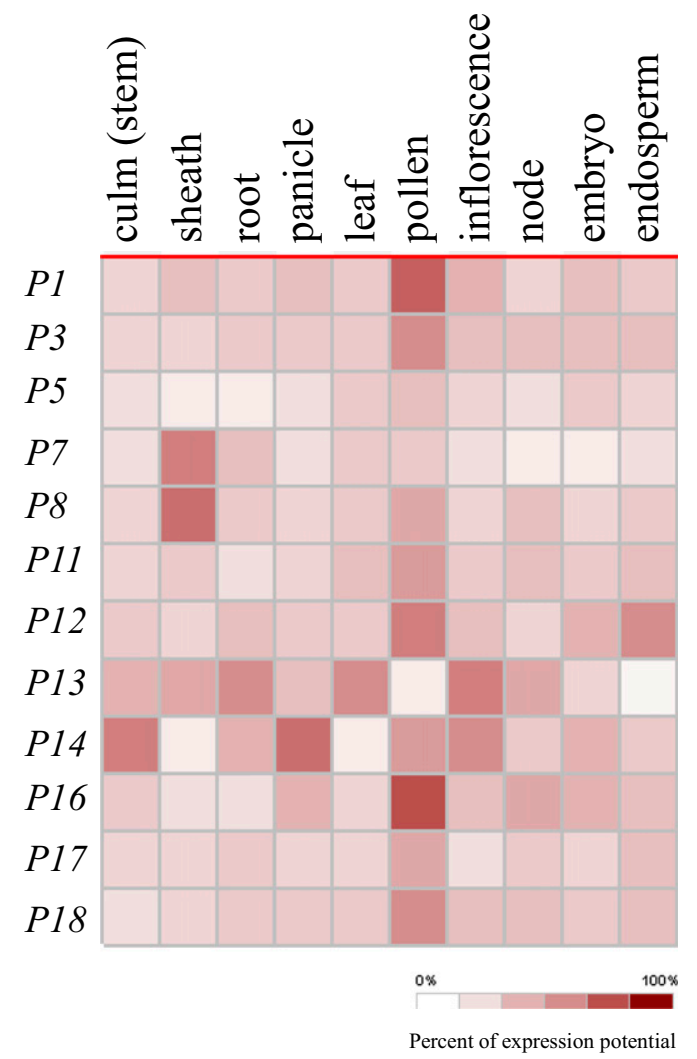

Fig. 5. Tissue-specific expression profiles of candidate genes based on microarray data from the GENEVESTIGATOR (https://genevestigator.com/gv/). resistance (Cesari et al. 2013; Jia et al. 2000; Kanzaki et al. 2012; Li et al. 2009a, b; Zhai et al. 2014), and only Pi-ta was reported as a race-specific $R$ gene (Lee et al. 2009a). In this research, seven isolates belonging to races 2011-40(E3), 2009-70-1(A7), 2010-491(B1), 2010-28(C1), 2010-42-2(F1), 2010-35-1(D13), and 20109 (G1) were inoculated in RIL populations, and it was found that only blast isolate $2010-9(\mathrm{G} 1)$ could be controlled by $P i-h k 2$. This suggested that $P i-h k 2$ might confer race-specific resistance to predominant isolate 2010-9 (G1) in Jiangsu Province, China.

Gene $P i-h k 2$ conferring resistance to the blast in landrace Heikezijing was mapped into an interval of $143 \mathrm{~kb}$ between ILP-19 and RM24048 on chromosome 9. It has been reported that three blast $R$ genes (Pi5/Pi3/Pi-i, Pi-15, and Pi56) are located near this region (Liu et al. 2013b). In order to distinguish $P i$ - $h k 2$ from them, an integrated physical map with these $R$ genes and linked markers were constructed base on the Nipponbare sequence (Fig. 7). Three linked markers (C1473, RM240044, and CRG5) were used to amplify the genomic DNA of Heikezijing and Suyunuo. No PCR product was detected by C1473 and RM24044 in Heikezijing and Suyunuo, and no fragment difference was detected by CRG5 in Heikezijing and Suyunuo. Otherwise, the whole gene sequences of Pi56, Pi5-1, and Pi5-2 genes were amplified from Heikezijing and Suyunuo, and the sequence alignments showed no differences of these three genes' sequences between Heikezijing and Suyunuo. In this map, the Pi-hk2 locus defined by ILP-19 and RM24048 was different from the loci of the other three genes. All of these results suggested that $P i$ - $h k 2$ might be a new blast $R$ gene.

There were two main references about disease rating systems. Mackill and Bonman (1992) showed that 0-to-5 scales were decided by lesion type and lesion size. Silué et al. (1992) showed a picture with different lesion type, lesion size, and lesion number in a 1-to-6 scale. In our manuscript, we referred these two articles and made a modification as described as Shi et al. (2010). In our scoring system, scores from 0 to 5 were based on lesion type and lesion size.

More than $70 R$ genes from different plant species have been cloned and characterized, and most of them encode NBS-LRR proteins (Liu et al. 2007a). Among 24 rice blast $R$ genes, 22 genes encode NBS-LRR proteins, except for $\mathrm{Pi}-\mathrm{d} 2$ encoding a B-lectin receptor kinase (Chen et al. 2006) and pi2l encoding a proline-rich protein that includes a putative heavy metal-binding domain and putative protein-protein interaction motifs (Fukuoka et al. 2009). In all, 18 putative genes were predicted in the target $143-\mathrm{kb}$ region conferring Pi-hk2 in the Nipponbare sequence. Among these

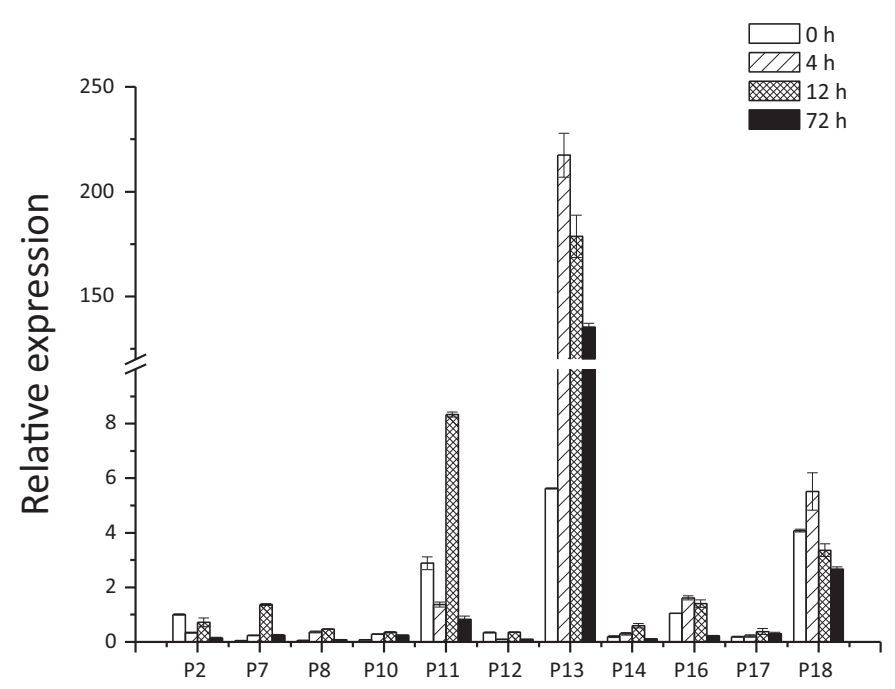

Fig. 6. Real-time polymerase chain reaction analysis for the expression patterns of candidate genes in Heikezijing induced by inoculation isolate 2010-9(G1); 18S-rRNA was used as an internal control. Data represent means and standard errors of three replicates. 


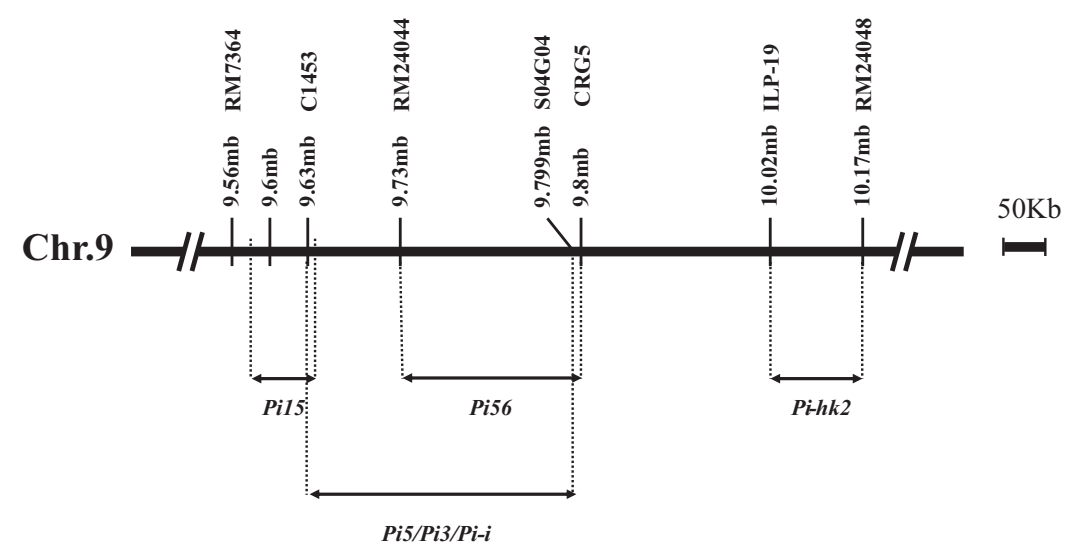

Fig. 7. Integrated physical mapping of four resistance genes (Pi15, Pi5/Pi3/Pi-i, Pi56, and Pi-hk2) which were mapped on chromosome 9.Positions of the resistance genes and markers were integrated on the basis of the data from the following references: Pi15 (Lin et al. 2007); Pi5/Pi3/Pi-i (Jeon et al. 2003; Lee et al.2009b; Yi et al. 2004); Pi56 (Liu et al. 2013b), and Pi-hk2 (this article). Markers used in this study and their physical positions are located on the top of the chromosome.

candidate genes, seven genes $(P 2, P 4, P 5, P 6, P 10, P 11$, and $P 15)$ encode transposon/retrotransposon proteins and only six genes $(P 7$, $P 8, P 13, P 14, P 16$, and $P 17$ ) encoded for function proteins. $P 7$ and $P 8$ encoded pleiotropic drug resistance protein 4 . However, the sequence similarity of $P 7$ and $P 8$ was low, and they may not have generated by duplication. $P 13$ encodes a WRKY transcription factor WRKY74, with conserved motif WRKYGQK at its $\mathrm{N}$ terminus. WRKY transcription factors generally act as transcriptional regulators through $\mathrm{W}$ boxes, cis-elements that are enriched in the promoters of several defense-related genes. It binds specifically to the DNA sequence motif (T)(T)TGAC(C/T), which is known as the $\mathrm{W}$ box. P14 encoded cytochrome b5-like heme/steroid-binding domain containing protein. The genome of Arabidopsis thaliana has 15 proteins with a cytochrome b5-like heme/steroid binding domain, and 1 of them acted as a regulator positively controlling LR formation, and functions independently of the ARF7/19-mediated auxin signaling pathway (Ikeyama et al. 2010). P16 encoded protein kinase, which played a role in a multitude of cellular processes, including division, proliferation, apoptosis, and differentiation. P17 encoded ankyrin repeat family protein, which is one of the most common protein-protein interaction motifs in nature. Li and Wang (2013) reported that, among the 13 rice WRKY genes, 11 genes are involved in the interaction of rice with $M$. oryzae, which causes blast diseases not only in rice but also in barley. Compared with other candidate genes, $P 11, P 13$, and $P 18$ were expressed higher based on the result of real-time PCR analysis in Heikezijing induced by inoculation with 2010-9(G1). According to these results, it appeared that $P 13$ and $P 18$ were more possibly related to rice blast resistance and might be the candidate genes of Pi-hk2. Among these predicted genes, none of these genes encoded NBSLRR protein, and our future work will focus on fine mapping and identifying the gene $P i-h k 2$.

\section{ACKNOWLEDGMENTS}

This research has been supported by grants from the National Key Project for Transgenic Crops (2016ZX08009-003-001 and 2014ZX08009-001B), the Natural Science Foundation of China (30900888,31171516), the Fundamental Research Funds for the Central Universities (KYZ201302), Jiangsu Agriculture Science, and the Technology Innovation fund (CX(12) 1003-3, CX(15)1054). We thank Z.-Y. Chen and Y.-F. Liu for kindly providing all of the blast isolates.

\section{LITERATURE CITED}

Ahn, S. N., Kim, Y. K., Hong, H. C., Han, S. S., Kwon, S. J., Choi, H. C., Moon, H. P., and McCouch, S. R. 2000. Molecular mapping of a new gene for resistance to rice blast (Pyricularia grisea Sacc.). Euphytica 116:17-22.
Ashikawa, I., Hayashi, N., Yamane, H., Kanamori, H., Wu, J. Z., Matsumoto, T., Ono, K., and Yano, M. 2008. Two adjacent nucleotide-binding siteleucine-rich repeat class genes are required to confer $P i k m$-specific rice blast resistance. Genetics 180:2267-2276.

Campbell, M. A., Chen, D., and Ronald, P. C. 2004. Development of codominant amplified polymorphic sequence markers in rice that flank the Magnaporthe grisea resistance gene Pi7 (t) in recombinant inbred line 29. Phytopathology 94:302-307.

Cesari, S., Thilliez, G., Ribot, C., Chalvon, V., Michel, C., Jauneau, A., Rivas, S., Alaux, L., Kanzaki, H., Okuyama, Y., Morel, J. B., Fournier, E., Tharreau, D., Terauchi, R., and Kroi, T. 2013. The rice resistance protein pair RGA4/RGA5 recognizes the Magnaporthe oryzae effectors AVR-Pia and AVR1-CO39 by direct binding. Plant Cell 25:1463-1481.

Chen, D. H., Viña, M. d., Inukai, T., Mackill, D. J., Ronald, P. C., and Nelson, R. J. 1999. Molecular mapping of the blast resistance gene, $P i 44(t)$, in a line derived from a durably resistant rice cultivar. Theor. Appl. Genet. 98: 1046-1053.

Chen, J., Peng, P., Tian, J. S., He, Y. G., Zhang, L. P., Liu, Z. X., Yin, D. D., and Zhang, Z. H. 2015. Pike, a rice blast resistance allele consisting of two adjacent NBS-LRR genes, was identified as a novel allele at the Pik locus. Mol. Breed. 35:117.

Chen, X., Shang, J., Chen, D., Lei, C., Zou, Y., Zhai, W., Liu, G., Xu, J., Ling, Z., Cao, G., Ma, B., Wang, Y., Zhao, X., Li, S., and Zhu, L. 2006. A B-lectin receptor kinase gene conferring rice blast resistance. Plant J. 46:794-804.

Dean, R. A., Talbot, N. J., Ebbole, D. J., Farman, M. L., Mitchell, T. K., Orbach, M. J., Thon, M., Kulkarni, R., Xu, J. R., Pan, H., Read, N. D., Lee, Y. H., Carbone, I., Brown, D., Oh, Y. Y., Donofrio, N., Jeong, J. S., Soanes, D. M., Djonovic, S., Kolomiets, E., Rehmeyer, C., Li, W., Harding, M., Kim, S., Lebrun, M. H., Bohnert, H., Coughlan, S., Butler, J., Calvo, S., Ma, L. J., Nicol, R., Purcell, S., Nusbaum, C., Galagan, J. E., and Birren, B. W. 2005. The genome sequence of the rice blast fungus Magnaporthe grisea. Nature 434:980-986.

Deng, Y. W., Zhu, X. D., Shen, Y., and He, Z. H. 2006. Genetic characterization and fine mapping of the blast resistance locus Pigm(t) tightly linked to $P i 2$ and $P i 9$ in a broad-spectrum resistant Chinese variety. Theor. Appl. Genet. 113:705-713.

DeYoung, B. J., and Innes, R. W. 2006. Plant NBS-LRR proteins in pathogen sensing and host defense. Nat. Immunol. 7:1243-1249.

Fjellstrom, R., Conaway-Bormans, C. A., McClung, A. M., Marchetti, M. A., Shank, A. R., and Park, W. D. 2004. Development of DNA markers suitable for marker assisted selection of three $P i$ genes conferring resistance to multiple Pyricularia grisea pathotypes. Crop Sci. 44:1790-1798.

Fukuoka, S., Saka, N., Koga, H., Ono, K., Shimizu, T., Ebana, K., Hayashi, N., Takahashi, A., Hirochika, H., Okuno, K., and Yano, M. 2009. Loss of function of a proline-containing protein confers durable disease resistance in rice. Science 325:998-1001.

Hayashi, K., and Yoshida, H. 2009. Refunctionalization of the ancient rice blast disease resistance gene Pit by the recruitment of a retrotransposon as a promoter. Plant J. 57:413-425.

He, X. Y., Liu, X. Q., Wang, L., Wang, L., Lin, F., Cheng, Y. S., Chen, Z. M., Liao, Y. P., and Pan, Q. H. 2012. Identification of the novel recessive gene pi55(t) conferring resistance to Magnaporthe oryzae. Sci. China Life Sci. 55:141-149.

Hua, L., Wu, J., Chen, C., Wu, W., He, X., Lin, F., Wang, L., Ashikawa, I., Matsumoto, T., Wang, L., and Pan, Q. 2012. The isolation of Pil, an allele 
at the Pik locus which confers broad spectrum resistance to rice blast. Theor. Appl. Genet. 125:1047-1055.

Huang, J., Wang, M. M., Jiang, Y., Bao, Y. M., Huang, X., Sun, H., Xu, D. Q., Lan, H. X., and Zhang, H. S. 2008. Expression analysis of rice A20/AN1type zinc finger genes and characterization of ZFP177 that contributes to temperature stress tolerance. Gene 420:135-144.

Ikeyama, Y., Tasaka, M., and Fukaki, H. 2010. RLF, a cytochrome b5-like heme/steroid binding domain protein, controls lateral root formation independently of ARF7/19-mediated auxin signaling in Arabidopsis thaliana. Plant J. 62:865-875.

Jeon, J. S., Chen, D., Yi, G. H., Wang, G. L., and Ronald, P. C. 2003. Genetic and physical mapping of $\mathrm{Pi5}(t)$, a locus associated with broad-spectrum resistance to rice blast. Mol. Genet. Genomics 269:280-289.

Jia, Y., McAdams, S. A., Bryan, G. T., Hershey, H. P., and Valent, B. 2000. Direct interaction of resistance gene and avirulence gene products confers rice blast resistance. EMBO J. 19:4004-4014.

Kanzaki, H., Yoshida, K., Saitoh, H., Fujisaki, K., Hirabuchi, A., Alaux, L., Fournier, E., Tharreau, D., and Terauchi, R. 2012. Arms race co-evolution of Magnaporthe oryzae AVR-Pik and rice Pik genes driven by their physical interactions. Plant J. 72:894-907.

Lander, E., and Kruglyak, L. 1995. Genetic dissection of complex traits: Guidelines for interpreting and reporting linkage results. Nat. Genet. 11: 241-247.

Lander, E. S., Green, P., Abrahamson, J., Barlow, A., Daly, M. J., Lincoln, S. E., Newberg, L. A., and Newburg, L. 1987. MAPMAKER: An interactive computer package for constructing primary genetic linkage maps of experimental and natural populations. Genomics 1:174-181.

Lee, S., Costanzo, S., Jia, Y., Olsen, K. M., and Caicedo, A. L. 2009a. Evolutionary dynamics of the genomic region around the blast resistance gene $P i$-ta in AA genome Oryza species. Genetics 183:1315-1325.

Lee, S.-K., Song, M.-Y., Seo, Y.-S., Kim, H.-K., Ko, S., Cao, P.-J., Suh, J.-P., Yi, G., Roh, J.-H., Lee, S., An, G., Hahn, T.-R., Wang, G.-L., Ronald, P., and Jeon, J.-S. 2009b. Rice Pi5-mediated resistance to Magnaporthe oryzae requires the presence of two CC-NB-LRR genes. Genetics 181:1627-1638.

Li, D., Wang, L., Wang, M., Xu, Y. Y., Luo, W., Liu, Y. J., Xu, Z. H., Li, J., and Chong, K. 2009a. Engineering OsBAK1 gene as a molecular tool to improve rice architecture for high yield. Plant Biotechnol. J. 7:791-806.

Li, H., and Wang, S. 2013. Disease resistance. Pages 161-175 in: Plant Genetics and Genomics: Crops and Models. Genetics and Genomics of Rice. Q. Zhang and R. A. Wing, eds. Springer, Heidelberg, Germany.

Li, H., and Ye, G. J. 2007. A modified algorithm for the improvement of composite interval mapping. Genetics 175:361-374.

Li, W., Wang, B. H., Wu, J., Lu, G. D., Hu, Y. J., Zhang, X., Zhang, Z. G., Zhao, Q., Feng, Q., Zhang, H. Y., Wang, Z. Y., Wang, G. L., Han, B., Wang, Z. H., and Zhou, B. 2009b. The Magnaporthe oryzae avirulence gene AvrPiz-t encodes a predicted secreted protein that triggers the immunity in rice mediated by the blast resistance gene Piz-t. Mol. Plant-Microbe Interact. 22: 411-420.

Lin, F., Liu, Y., Wang, L., Liu, X., and Pan, Q. H. 2007. A high-resolution map of the rice blast resistance gene Pil5 constructed by sequence-ready markers. Plant Breed. 126:287-290.

Liu, B., Zhang, S., Zhu, X., Yang, Q., Wu, S., Mei, M., Mauleon, R., Leach, J., Mew, T., and Leung, H. 2004. Candidate defense genes as predictors of quantitative blast resistance in rice. Mol. Plant-Microbe Interact. 17:1146-1152.

Liu, J. L., Liu, X. L., Dai, L. Y., and Wang, G. L. 2007a. Recent progress in elucidating the structure, function and evolution of disease resistance genes in plants. J. Genet. Genomics 34:765-776.

Liu, W., Liu, J., Ning, Y., Ding, B., Wang, X., Wang, Z., and Wang, G. L. 2013a. Recent progress in understanding PAMP- and effector-triggered immunity against the rice blast fungus Magnaporthe oryzae. Mol. Plant 6:605-620.

Liu, X. Q., Lin, F., Wang, L., and Pan, Q. H. 2007b. The in silico map-based cloning of Pi36, a rice coiled-coil-nucleotide-binding site-leucine-rich repeat gene that confers race-specific resistance to the blast fungus. Genetics 176:2541-2549.

Liu, Y., Liu, B., Zhu, X. Y., Yang, J. Y., Bordeos, A., and Wang, G. L. 2013b. Fine-mapping and molecular marker development for Pi56(t), a NBS-LRR gene conferring broad-spectrum resistance to Magnaporthe oryzae in rice. Theor. Appl. Genet. 126:985-998.

Livak, K. J., and Schmittgen, T. D. 2001. Analysis of relative gene expression data using real-time quantitative PCR and the $2^{-\Delta \Delta C T}$ method. Methods 25: 402-408.

Luck, J. E., Lawrence, G. J., Dodds, P. N., Shepherd, K. W., and Ellis, J. G. 2000. Regions outside of the leucine-rich repeats of flax rust resistance proteins play a role in specificity determination. Plant Cell 12:1367-1377.

Ma, J., Lei, C., Xu, X., Hao, K., Wang, J., Cheng, Z., Ma, X., Ma, J., Zhou, K., and Zhang, X. 2015. Pi64, encoding a novel CC-NBS-LRR protein, confers resistance to leaf and neck blast in rice. Mol. Plant-Microbe Interact. 28: 558-568

Mackill, D. J., and Bonman, J. M. 1992. Inheritance of blast resistance in nearisogenic lines of rice. Phytopathology 82:746-749.

McCouch, S. R., Teytelman, L., Xu, Y., Lobos, K. B., Clare, K., Walton, M., Fu, B., Maghirang, R., Li, Z., and Xing, Y. 2002. Development and mapping of 2240 new SSR markers for rice (Oryza sativa L.). DNA Res. 9: 199-207.

Pich, U., and Schubert, I. 1993. Midiprep method for isolation of DNA from plants with a high content of polyphenolics. Nucleic Acids Res. 21: 3328-3330.

Rairdan, G. J., Collier, S. M., Sacco, M. A., Baldwin, T. T., Boettrich, T., and Moffett, P. 2008. The coiled-coil and nucleotide binding domains of the Potato Rx disease resistance protein function in pathogen recognition and signaling. Plant Cell 20:739-751.

Sakata, K., Nagamura, Y., Numa, H., Antonio, B. A., Nagasaki, H., Idonuma, A., Watanabe, W., Shimizu, Y., Horiuchi, I., Matsumoto, T., Sasaki, T., and Higo, K. 2002. RiceGAAS: An automated annotation system and database for rice genome sequence. Nucleic Acids Res. 30:98-102.

Sharma, T. R., Rai, A. K., Gupta, S. K., Vijayan, J., Devanna, B. N., and Ray, S. 2012. Rice blast management through host-plant resistance: Retrospect and prospects. Agric. Res. 1:37-52.

Shi, X. L., Wang, J. F., Bao, Y. M., Li, P. F., Xie, L. J., Huang, J., and Zhang, H. S. 2010. Identification of the quantitative trait loci in japonica rice landrace Heikezijing responsible for broad-spectrum resistance to rice blast. Phytopathology 100:822-829.

Silué, D., Notteghem, J. L., and Tharreau, D. 1992. Evidence of a gene-forgene relationship in the Oryza sativa-Magnaporthe grisea pathosystem. Phytopathology 82:577-580.

Skamnioti, P., and Gurr, S. J. 2009. Against the grain: Safeguarding rice from rice blast disease. Trends Biotechnol. 27:141-150.

Tabien, R. E., Li, Z., Paterson, A. H., Marchetti, M. A., Stansel, J. W., Pinson, S. R. M., and Park, W. D. 2000. Mapping of four major rice blast resistance genes from 'Lemont' and 'Teqing' and evaluation of their combinatorial effect for field resistance. Theor. Appl. Genet. 101:1215-1225.

Wang, G. L., Mackill, D. J., Bonman, J. M., McCouch, S. R., Champoux, M. C., and Nelson, R. J. 1994. RFLP mapping of genes conferring complete and partial resistance to blast in a durably resistant rice cultivar. Genetics 4:1421-1434.

Wang, J. F., He, X. J., Zhang, H. S., and Chen, Z. Y. 2002. Genetic analysis of blast resistance in japonica rice landrace Heikezijing from Taihu region. Yi Chuan Xue Bao 29:803-807.

Wei, H., Fu, Y., and Arora, R. 2005. Intron-flanking EST-PCR markers: From genetic marker development to gene structure analysis in Rhododendron. Theor. Appl. Genet. 111:1347-1356.

Wu, Y. Y., Bao, Y. M., Xie, L. J., Su, Y. Y., Chu, R. Z., He, W. W., Huang, J., Wang, J. F., and Zhang, H. S. 2013. Fine mapping and identification of blast resistance gene $P i-h k l$ in a broad-spectrum resistant japonica rice landrace. Phytopathology 103:1162-1168.

Yi, G., Lee, S. K., Hong, Y. K., Cho, Y. C., Nam, M. H., Kim, S. C., Han, S. S., Wang, G. L., Hahn, T. R., and Ronald, P. C. 2004. Use of Pi5(t) markers in marker-assisted selection to screen for cultivars with resistance to Magnaporthe grisea. Theor. Appl. Genet. 109:978-985.

Yuan, B., Zhai, C., Wang, W. J., Zeng, X. S., Xu, X. K., Hu, H. Q., Lin, F., Wang, L., and Pan, Q. H. 2011. The Pik-p resistance to Magnaporthe oryzae in rice is mediated by a pair of closely linked CC-NBS-LRR genes. Theor. Appl. Genet. 122:1017-1028.

Zhai, C., Lin, F., Dong, Z. Q., He, X. Y., Yuan, B., Zeng, X. S., Wang, L., and Pan, Q. H. 2011. The isolation and characterization of Pik, a rice blast resistance gene which emerged after rice domestication. New Phytol. 189: 321-334.

Zhai, C., Zhang, Y., Yao, N., Lin, F., Liu, Z., Dong, Z. Q., Wang, L., and Pan, Q. H. 2014. Function and interaction of the coupled genes responsible for Pik- $h$ encoded rice blast resistance. PLoS One 9:e98067.

Zhou, B., Qu, S. H., Liu, G. F., Dolan, M., Sakai, H., Lu, G. D., Bellizzi, M., and Wang, G. L. 2006. The eight amino-acid differences within three leucine-rich repeats between $P i 2$ and Piz-t resistance proteins determine the resistance specificity to Magnaporthe grisea. Mol. Plant-Microbe Interact. 19:1216-1228

Zhu, M. L., Ling, W., and Pan, Q. H. 2004. Identification and characterization of a new blast resistance gene located on rice chromosome 1 through linkage and differential analyses. Phytopathology 94:515-519.

Zhu, X. Y., Chen, S., Yang, J. Y., Zhou, S. C., Zeng, L. X., Han, J. L., Su, J., Wang, L., and Pan, Q. H. 2012. The identification of Pi50(t), a new member of the rice blast resistance Pi2/Pi9 multigene family. Theor. Appl. Genet. 124:1295-1304. 